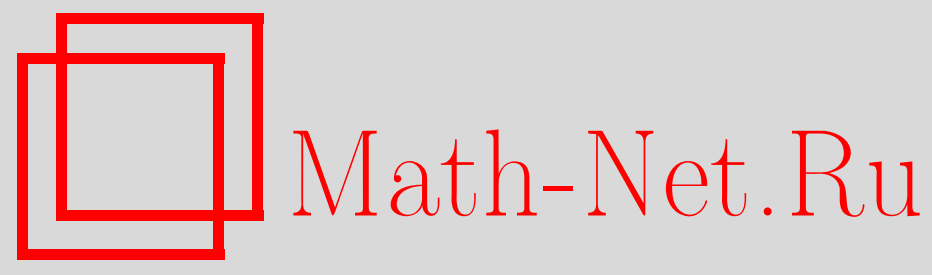

М. И. Войнова, Л. С. Ефремова, О динамике простейших отображений дендритов, Матем. заметки, 1998, том 63, выпуск 2, 183-195

DOI: https://doi.org/10.4213/mzm1265

Использование Общероссийского математического портала Math-Net.Ru подразумевает, что вы прочитали и согласны с пользовательским соглашением http://www . mathnet.ru/rus/agreement

Параметры загрузки:

IP : 54.80 .73 .141

26 апреля 2023 г., 10:56:06 


\title{
О ДИНАМИКЕ ПРОСТЕЙШИХ ОТОБРАЖЕНИЙ ДЕНДРИТОВ
}

\author{
М.И. Войнова, Л. С. Ефремова
}

\begin{abstract}
Введено понятие простейшего отображения дендрита в себя. Указаны арифметические соотношения между периодами периодических точек; установлена структура $\omega$-предельных множеств, множеств периодических и неблуждающих точек; показано, что топологическая энтропия простейших отображений равна 0. Приведены примеры, иллюстрирующие различия в энтропийных свойствах непрерывных отображений дендритов со счетным множеством точек ветвления и непрерывных отображений их ретрактов - конечных деревьев.
\end{abstract}

Библиография: 20 названий.

1. Введение. Непрерывным отображениям отрезка и окружности посвящена обширная литература. Так, в [1]-[10] рассматривались вопросы сосуществования периодов периодических точек, влияния арифметических соотношений между периодами на топологическую энтропию, изучалась структура неблуждающего множества. Одно из направлений естественного развития этих исследований связано с обобщением указанных результатов на случай непрерывных отображений конечных графов [11]-[15].

В настоящей заметке рассмотрен класс топологических пространств (дендритов), которые допускают не только конечное, но и счетное множество точек ветвления и обладают свойством дугообразной связности.

Сформулируем необходимые определения и напомним основные свойства дендритов (см. [16, гл. $6, \S 51])$.

ОПРЕДЕЛЕНИЕ 1. Дендритом назьвается локально связньй континуум (компактное связное метрическое пространство), не содержащий подмножеств, гомеоморфных окружности.

Под дугой в дендрите $X$ будем понимать множество, гомеоморфное промежутку на прямой. Одноточечноемножество отнесем к классу дуг, считая его вырожденной дугой.

Свойство 1 (дугообразная связность). Всякую пару точек $x \neq y$ в дендрите $X$ мохсно соединить единственной дугой, имеющей в качестве концов точки $х и$.

В дальнейшем символом $\gamma(x, y)$ будем обозначать дугу с концами в точках $x$ и $y$, содержащую эти точки.

Работа выполнена при поддержке Российского фонда фундаментальных исследований, грант № 96-01-01755. 
ОПРЕДЕЛЕНИЕ 2 . Пусть $X-$ произвольный континуум, $\mathfrak{n}-$ порядковое число $\omega$ множества целых неотрицательных чисел в их естественном порядке или кардинальное число $\leqslant c$ ( $c$ - мощность континуума). Говорят, что порядок точки $x$ не превосходит $\mathfrak{n}$ $(\operatorname{ord} x \leqslant \mathfrak{n})$, если для любого $\varepsilon>0$ существует $\delta$-окрестность $U_{\delta}(x)$ точки $x$ при $0<\delta<\varepsilon$ такая, что $\operatorname{card}\left(\partial U_{\delta}(x)\right) \leqslant \mathfrak{n}$, где $\partial(\cdot)$ - гранища множества, a card $(\cdot)$ - мощность множества. Равенство ord $x=\mathfrak{n}$ означает, что ord $x \leqslant \mathfrak{n}$ и соотношение ord $x \leqslant \mathfrak{m}$ не имеет места ни при каком $\mathfrak{m} \leqslant \mathfrak{n}$.

Свойство 2 (регулярность). Всякий дендрит $X$ есть регулярный континуум, т.е. любая точка $x \in X$ имеет порядок $\leqslant \omega$.

Таким образом, всякий подконтинуум дендрита есть дендрит. Дополним свойство регулярности следуюшими утверждениями.

Теорема 1. Пусть $X$-дендрит, содержсащий более одной точки. Тогда в $X$ существуют точки первого порядка, называемые концевыми; всюду плотны точки второго порядка; существует не более чем счетное множество точек ветвления (точек порядка большего 2).

В дальнейшем будем обозначать через $E(X)$ множество конщевых точек, а через $R(X)$ - множество точек ветвления дендрита $X$.

ТеОрема 2. Если $X$ - дендрит и для некоторой точки $x \in X$ число компонент множества $X \backslash\{x\}$ конечно, то это число совпадает $c \operatorname{ord} x$.

Свойство 3. Пусть $X-$ дендрит, а $Y$ - собственный подконтинуум $X$. Тогда любая компонента $U$ открытого множества $X \backslash Y$ имеет в $X$ единственную граничную точку $u \in Y(\partial U=u)$.

В настоящей заметке рассматривается класс $D$ дендритов, обладающих следующими свойствами: для любого $X \in D$

1) $R(X)$ - замкнутое множество;

2) каково бы ни было $x \in R(X)$, число компонент множества $X \backslash\{x\}$ конечно.

Из теорем 1,2 следует, что если $X \in D$ имеет точки ветвления, то $R(X)$ - разреженное множество, и для любого $x \in R(X)$ число компонент множества $X \backslash\{x\}$ совпадает $\operatorname{cord} x$, где ord $x \geqslant 3$.

Условимся обозначать через $X_{i}(x), 1 \leqslant i \leqslant \operatorname{ord} x$, компоненты множества $X \backslash\{x\}$, где $x$ - произвольная точка ветвления $X$. Приведем необходимые примеры дендритов из множества $D$.

Пример 1. $n$-од $X=\left\{z \in \mathbb{C}: z^{n} \in[0,1]\right\}$, где $\mathbb{C}$ - комплексная плоскость, $n \geqslant 3$, имеет единственную точку ветвления $O$, причем $\operatorname{ord} O=n$.

ПРИмеР 2. В пространстве $\mathbb{R}^{3}$, снабженном прямоугольной декартовой системой координат $O x_{1} x_{2} x_{3}$, укажем дендрит $X \in D$ со счетным множеством точек ветвления. Возьмем произвольную последовательность натуральных чисел $3 \leqslant n_{0} \leqslant n_{1}<\cdots<$ $n_{j}<\cdots$. Обозначим через $X_{0}^{\left(n_{j}\right)} n$-од в плоскости $x_{3}=0$, где $n=n_{j}, j \geqslant 0$. Положим

$$
X^{\left(n_{j}\right)}= \begin{cases}X_{0}^{\left(n_{0}\right)} & \text { при } j=0, \\ \left\{\left(\frac{x_{1}}{j}, \frac{x_{2}}{j}, \frac{1}{j}\right):\left(x_{1}, x_{2}, 0\right) \in X_{0}^{\left(n_{j}\right)}\right\} & \text { при } j \geqslant 1 .\end{cases}
$$


Определим дендрит

$$
X=X^{(0)} \cup \bigcup_{j=0}^{+\infty} X^{\left(n_{j}\right)}, \quad \text { где } X^{(0)}=\left\{\left(0,0, x_{3}\right): x_{3} \in[0,1]\right\} .
$$

Здесь $R(X)=\{(0,0,0)\} \cup\{(0,0,1 / j)\}_{j \geqslant 1}-$ замкнутое множество, $\{\operatorname{ord} x\}_{x \in R(X)}-$ неограниченное множество, a ord $O=n_{0}+1$.

2. Простейшие отображения. Свойства. Формулировки основных результатов. Введем понятие простейшего отображения дендрита в себя.

ОПРЕДЕЛЕНИЕ 3. Пусть $X$ - дендрит. Отображение $f: X \rightarrow X$ назовем простейuим, если $f$ непрерывно и полньй прообраз любой дуги из $f(X)$ есть дуга в $X$.

Обратим внимание на то, что каждое простейшее отображение является монотонным, в то время как для дендритов с непустым множеством точек ветвления существуют монотонные, но не простейшие, отображения (определение монотонного отображения произвольного связного топологического пространства см. в [16, с. 140]).

Обозначим через $P^{0}(X)$ множество всех простейших отображений дендрита $X \in D$ в себя. Каково бы ни было $f \in P^{0}(X)$, при любом $i \geqslant 1 f^{i} \in P^{0}(X)$. Укажем, что пример простейшего отображения доставляет поворот $n$-ода $X(n \geqslant 3)$ на угол $2 \pi / n$ с неподвижной точкой $O$.

Приведенные вьше определения позволяют сформулировать основные результаты работы. Произвольное непрерьвное отображение дендрита в себя имеет неподвижную точку [17]. Поэтому множество $\operatorname{Per}(f)$ периодических точек отображения $f \in P^{0}(X)$ непусто. Обозначим через $T(f)$ множество периодов периодических точек отображения $f \in P^{0}(X)$.

Tеорема 3. Пусть $f \in P^{0}(X)$, a $T(f)$ - неограниченное мнохество. Тогда множество $R(X)$ точек ветвления $X$ счетно.

Наряду со структурой множества периодических точек мы опишем структуру $\omega$-предельного множества $\omega(x, f)$ траектории произвольной точки $x \in X$, множества неблуждаюших точек $\Omega(f)$ и топологическую энтропию $h(f)$ отображения $f \in P^{0}(X)$.

TeOpema 4. Пусть $f \in P^{0}(X)$. Тогда

1) мнохсество $\operatorname{Per}(f)$ замкнуто;

2) для любого $x \in X \quad \omega(x, f)$ - периодическая орбита;

3) $\Omega(f)=\bigcup_{x \in X} \omega(x, f)=\operatorname{Per}(f)$;

4) $h(f)=0$.

ТеОрема 5. 1) Каково бы ни было натуральное число $m \geqslant 1$, существуют дендрит $X \in D$ со счетным множсеством точек ветвления и непрерывное отображение $f: X \rightarrow X$ такое, что

$$
h(f)=0, \quad T(f)=\{1, m, 2 m, \ldots, j m, \ldots\}, \quad j \geqslant 1 .
$$

2) Каково бы ни было простое число $p$, существуют дендрит $X \in D$ со счетным мнохеством точек ветвления и непрерывное отобрахсение $f: X \rightarrow X$ такое, чmo

$$
h(f)=0, \quad T(f)=\left\{p^{j}\right\}_{j \geqslant 0} .
$$


Теорема 5 выявляет принципиальные различия в свойствах непрерывных отображений дендритов со счетным множеством точек ветвления и непрерывных отображений их ретрактов - конечных деревьев. Так, произвольное непрерывное отображение конечного дерева в себя имеет множество $T(f)$, содержащее натуральные числа вида $\{m j\}$ при некотором $m \geqslant 1$ и любом $j \geqslant 1$, в том и только том случае, когда $h(f)>0$ [15].

Вопрос Ллибре-Мисюревича о существовании непрерьвньх отображений, заданных на конечных деревьях, имеющих нулевую топологическую энтропию и множество периодов периодических точек вида $\left\{p^{j}\right\}_{j} \geqslant 0$, где $p$ - простое число [14], в настоящее время остается открытым.

Для доказательства теорем 3-5 потребуется ряд свойств простейших отображений.

Лемма 1. Если $f \in P^{0}(X)$, то для любых точек $x, y \in X$ выполнено равенство $f(\gamma(x, y))=\gamma(f(x), f(y))$.

ДокАЗАТЕльство. Утверждение леммы 1 справедливо, если $Y=f(\gamma(x, y))$ одноточечное множество. Предположим, что $Y$ содержит более одной точки. Тогда $\gamma(x, y)$ - невырожденная дуга. Из компактности $X$, непрерывности $f$ и свойства 2 следует, что $Y$ - дендрит. Используя определение 3 и свойство 1 дугообразной связности $Y$, немедленно получаем, что если существует точка $z \in \gamma(x, y) \backslash\{x, y\}$ такая, что $f(z) \in E(Y)$, то выполнено одно из равенств: $f(z)=f(x)$ или $f(z)=f(y)$. Таким образом, $\operatorname{card} E(Y)=2$, и $Y=\gamma(f(x), f(y))$. Лемма 1 доказана.

Возьмем произвольную периодическую точку $x^{0}$ периода $m \geqslant 1$ отображения $f \in$ $P^{0}(X)$. Обозначим через $\operatorname{Orb}\left(x^{0}, f\right)$ периодическую орбиту, порожденную точкой $x^{0}$ :

$$
\operatorname{Orb}\left(x^{0}, f\right)=\left\{x^{0}, \ldots, x^{m-1}\right\}, \quad \text { где } \quad x^{i}=f^{i}\left(x^{0}\right), \quad 0 \leqslant i \leqslant m-1 .
$$

СледСтвиЕ 1. Пусть $f \in P^{0}(X), x^{0} \in \operatorname{Per}(f), m-$ период $x^{0}$. Тогда следующие утверждения әквивалентны:

1) существует дуга в $X$, содержащая $\operatorname{Orb}\left(x^{0}, f\right)$;

2) $m=1$ или 2 .

СлЕДСТВИЕ 2. Если $f \in P^{0}(X)$, то $R(X)$ - инвариантное множество.

СЛЕДСТВИЕ 3. Пусть $f \in P^{0}(X)$, а дендрит $X$ гомеоморфен $n$-оду при некотором $n \geqslant 3$. Тогда единственная точка ветвления $X$ является неподвижной точкой $f$.

Лемма 2. Пусть $f \in P^{0}(X), a R(X) \neq \varnothing$. Тогда $R(X) \cap \operatorname{Per}(f) \neq \varnothing$.

ДокАЗАТЕЛЬСтво. Инвариантность и замкнутость непустого множества $R(X)$ влекут справедливость включения $\omega(x, f) \subset R(X)$ для $\omega$-предельного множества траектории произвольной точки $x \in R(X)$. Так как $R(X)$ не более чем счетно, получаем, что и $\omega(x, f)$ не более чем счетно. Тогда в силу [18] $\omega(x, f) \cap \operatorname{Per}(f) \neq \varnothing$. Лемма 2 доказана.

СледСтвиЕ 4. Пусть $f \in P^{0}(X), z^{0} \in R(X) \cap \operatorname{Per}(f), m \geqslant 1-$ период $z^{0}$. Если для некоторой точки $x^{0} \in X_{i_{1}}\left(z^{0}\right)$ справедливо $f^{m}\left(x^{0}\right) \in X_{i_{2}}\left(z^{0}\right)\left(i_{1}, i_{2}-\right.$ произвольные натуральные числа, $1 \leqslant i_{1}, i_{2} \leqslant$ ord $\left.z^{0}\right)$, то для любой точки $x \in \overline{X_{i_{1}}\left(z^{0}\right)}$ выполнено $f^{m}(x) \in \overline{X_{i_{2}}\left(z^{0}\right)}$, здесь черта означает замыкание множества. 
ДокАЗАТЕльСТво. Предположим, что найдутся компонента $X_{i_{1}}\left(z^{0}\right), 1 \leqslant i_{1} \leqslant$ ord $z^{0}$, и различные точки $x^{0}, x \in X_{i_{1}}\left(z^{0}\right)$ такие, что

$$
f^{m}\left(x^{0}\right) \in X_{i_{2}}\left(z^{0}\right), \quad f^{m}(x) \in X_{i_{3}}\left(z^{0}\right), \quad \text { где } i_{2} \neq i_{3}, \quad 1 \leqslant i_{2}, i_{3} \leqslant \text { ord } z^{0} .
$$

Тогда $z^{0} \in \gamma\left(f^{m}\left(x^{0}\right), f^{m}(x)\right)=f^{m}\left(\gamma\left(x^{0}, x\right)\right)$. Поэтому полный прообраз $f^{-m}\left(z^{0}\right)$ точки $z^{0}$, наряду с $z^{0}$, содержит и некоторую точку $z \in \gamma\left(x^{0}, x\right)$. Так как невырожденная дуга $\gamma\left(z^{0}, z\right)$ содержит одну из точек $x$ или $x^{0}$, из (1) и свойства дугообразной связности дендрита $X$ следует, что $f^{-m}\left(z^{0}\right)$ не является дугой. Полученное противоречие с $f \in P^{0}(X)$ доказывает следствие 4 .

Аналогично следствию 4 доказывается

СледСтвие 5. Пусть $f \in P^{0}(X), z^{0} \in R(X) \cap \operatorname{Per}(f), m \geqslant 1-$ nериод $z^{0}$. Тогда для любых различных $i_{1}, i_{2}, 1 \leqslant i_{1}, i_{2} \leqslant$ ord $z^{0}$, справедливо

$$
f^{m}\left(\overline{X_{i_{1}}\left(z^{0}\right)}\right) \cap f^{m}\left(\overline{X_{i_{2}}\left(z^{0}\right)}\right)=\left\{z^{0}\right\}
$$

Пусть $\gamma\left(z^{0}, y^{0}\right)$ - произвольная невырожденная дуга в дендрите $X, \varphi:[0,1] \rightarrow \gamma\left(z^{0}, y^{0}\right)$ - гомеоморфизм. Тогда $\varphi$ порождает на $\gamma\left(z^{0}, y^{0}\right)$ отношение порядка “२ " $(\varphi$-предшествует).

ОПРЕДЕЛЕНИЕ 4. Каковы бы ни были различные точки $x^{\prime}, x^{\prime \prime} \in \gamma\left(z^{0}, y^{0}\right)$, будем говорить, что $x^{\prime} \prec_{\varphi} x^{\prime \prime}$, если $\varphi^{-1}\left(x^{\prime}\right)<\varphi^{-1}\left(x^{\prime \prime}\right)$, где “<" - естественное отношение порядка на отрезке [0, 1].

СлеДСТВИе 6. Пусть $f \in P^{0}(X), z^{0} \in R(X) \cap \operatorname{Per}(f), m \geqslant 1-$ nериод $z^{0}$. Тогда каковы бы ни были компонента $X_{i}\left(z^{0}\right)$ множества $X \backslash\left\{z^{0}\right\}, 1 \leqslant i \leqslant \operatorname{ord} z^{0}, u$ периодическая точка $x^{0} \notin \operatorname{Orb}\left(z^{0}, f\right)$, любая невырожсенная дуга $\gamma\left(z^{0}, z\right) \subset \overline{X_{i}\left(z^{0}\right)}$ содержит не более одной точки периодической орбиты $\operatorname{Orb}\left(x^{0}, f^{m}\right)$.

ДокАЗАТЕЛЬСТво. Предположим, что существуют компонента $X_{i}\left(z^{0}\right)$ множества $X \backslash\left\{z^{0}\right\}, 1 \leqslant i \leqslant \operatorname{ord} z^{0}$, периодическая точка $x^{0} \notin \operatorname{Orb}\left(z^{0}, f\right)$ и невырожденная дуга $\gamma\left(z^{0}, z\right) \subset \overline{X_{i}\left(z^{0}\right)}$ такая, что $\gamma\left(z^{0}, z\right)$ содержит не менее двух точек периодической орбиты $\operatorname{Orb}\left(x^{0}, f^{m}\right)$. Пусть точка $y^{0} \in \operatorname{Orb}\left(x^{0}, f^{m}\right) \cap \gamma\left(z^{0}, z\right)$ такова, что при некотором $m_{0} \geqslant 1 y^{m m_{0}}=f^{m m_{0}}\left(y^{0}\right) \in \gamma\left(z^{0}, y^{0}\right) \backslash\left\{z^{0}, y^{0}\right\}$, а гомеоморфизм $\varphi:[0,1] \rightarrow \gamma\left(z^{0}, y^{0}\right)$ удовлетворяет следующим условиям: $\varphi(0)=z^{0}, \varphi(1)=y^{0}$. Тогда в силу леммы 1 и определения 4 дуга $\gamma\left(z^{0}, y^{0}\right)$ инвариантна относительно $f^{m m_{0}}$ и

$$
z^{0} \prec_{\varphi} \cdots \prec_{\varphi} y^{(j+1) m m_{0}} \prec_{\varphi} y^{j m m_{0}} \prec_{\varphi} \cdots \prec_{\varphi} y^{m m_{0}} \prec_{\varphi} y^{0}, \quad \text { где } j \geqslant 1 \text {. }
$$

Последнее противоречит тому, что $y^{0} \in \operatorname{Per}(f)$. Следствие 6 доказано.

3. Сосуществование периодов периодических точек простейших отображений. Доказательство теоремы 3. Мы начнем с рассмотрения арифметических соотношений между периодами периодических точек простейших отображений $n$-ода $X$ $(n \geqslant 3)$. Занумеруем каким-либо образом компоненты множества $X \backslash\{O\}$ (например, обозначив через $X_{i}$ ту компоненту множества $X \backslash\{O\}$, точки которой имеют угловую координату $2 \pi(i-1) / n, 1 \leqslant i \leqslant n)$.

В силу следствий 3 и 6 справедлива 
Лемма 3. Пусть $f \in P^{0}(X)$, где $X-n$-од $(n \geqslant 3)$. Тогда для любой периодической орбиты $\operatorname{Orb}\left(x^{0}, f\right)$ периода $m>1$ выполнено неравенство $m \leqslant n$, причем точки $\operatorname{Orb}\left(x^{0}, f\right)$ располагаются на $m$ компонентах мнохества $X \backslash\{O\}$.

Для произвольной периодической орбиты $\operatorname{Orb}\left(x^{0}, f\right)$ периода $m>1$ введем в рассмотрение индексное множество

$$
I\left(\operatorname{Orb}\left(x^{0}, f\right)\right)=\left\{i: \operatorname{Orb}\left(x^{0}, f\right) \cap X_{i} \neq \varnothing, 1 \leqslant i \leqslant n\right\} .
$$

Из леммы 3 следует, что card $I\left(\operatorname{Orb}\left(x^{0}, f\right)\right)=m$.

В силу следствий 4,6 справедлива

Лемма 4. Пусть $X-n$-од $(n \geqslant 3), \operatorname{Orb}\left(x^{0}, f\right)$ u $\operatorname{Orb}\left(x_{1}^{0}, f\right)-$ периодические орбиты отображсеня $f \in P^{0}(X)$ периодов $m_{1}$ и $m_{2}$ соответственно, причем $m_{1} \neq m_{2}, m_{1}, m_{2}>1$. Тогда $I\left(\operatorname{Orb}\left(x^{0}, f\right)\right) \cap I\left(\operatorname{Orb}\left(x_{1}^{0}, f\right)\right)=\varnothing$.

Определим модельное отображение $\tau: X \rightarrow X$, которое будем называть связкой поворотов $n$-ода $X(n \geqslant 3)$. Для этого зададим произвольно $r$ натуральньх чисел $m_{j}$, $1 \leqslant j \leqslant r$, так чтобы $\sum_{j=1}^{r} m_{j}=n$. Разобьем $X$ на $r$ подмножеств

$$
L^{(j)}=\bigcup_{i=1}^{m_{j}} \overline{X_{i}^{(j)}}, \quad 1 \leqslant j \leqslant r
$$

где компоненты $X_{1}^{(j)}, X_{2}^{(j)}, \ldots, X_{m_{j}}^{(j)}$ множества $X \backslash\{O\}$ попарно различны, и

$$
L^{\left(j_{1}\right)} \cap L^{\left(j_{2}\right)}=\{O\}, \quad j_{1} \neq j_{2}, \quad 1 \leqslant j_{1}, j_{2} \leqslant r .
$$

Потребуем, чтобы при любом $x \in X$ выполнялось равенство $\rho(x, O)=\rho(\tau(x), O)$, где $\rho$ - метрика в $X$. При каждом $j, 1 \leqslant j \leqslant r$, положим $\tau\left(\overline{X_{m_{j}}^{(j)}}\right)=\overline{X_{1}^{(j)}}$. Если $m_{j}>1$, то каково бы ни было $i, 1 \leqslant i \leqslant m_{j}-1$, положим $\tau\left(\overline{X_{i}^{(j)}}\right)=\overline{X_{i+1}^{(j)}}$.

Подчеркнем, что $\tau \in P^{0}(X)$ однозначно определяется разбиением множества компонент $X \backslash\{O\}$ на $r$ подмножеств $\left\{X_{1}^{(j)}, \ldots, X_{m_{j}}^{(j)}\right\}_{j=1}^{r}$.

ОПредЕЛЕниЕ 5. Конечное упорядоченное множество $M$, элементами которого являются натуральные числа, будем назьвать допустимым, если существует простейшее отображение $n$-ода $X(n \geqslant 3)$ в себя, множество периодов периодических точек которого совпадает с $M$.

Введение понятия допустимого множества позволяет свести задачу сосуществования периодов периодических точек простейших отображений $n$-ода к задаче описания совокупности всех допустимых множеств. В такой постановке задача сосуществования периодов периодических точек непрерывных отображений $n$-ода ранее не рассматривалась (ср., например, с [11], [13]).

ТЕОрема 6. Конечное упорядоченное мнохсество $M=\left\{m_{1}, \ldots, m_{r}\right\}$, где $m_{1}<$ $\cdots<m_{r}$ при $r>1$, допустимо в том и только том случае, когда

$$
m_{1}=1 \quad u \quad \sum_{j=2}^{r} m_{j} \leqslant n \quad \text { npu } \quad r>1 .
$$


ДокАЗАТЕЛЬСтво. 1) Пусть множество $M$ допустимо, а отображение $f \in P^{0}(X)$ $(X-$ это $n$-од, $n \geqslant 3)$ таково, что $T(f)=M$. Тогда в силу следствия 3 , лемм 3 и 4 выполнено (2).

2) Пусть вьполнено (2). Покажем, что множество $M$, состоящее из натуральных чисел $m_{1}, \ldots, m_{r}\left(m_{1}<\cdots<m_{r}\right.$ при $\left.r>1\right)$ допустимо. Положим

$$
r^{*}=n-\sum_{j=2}^{r} m_{j}, \quad r^{*} \geqslant 0 .
$$

Построим разбиение $n$-ода $X(n \geqslant 3)$ на $r-1+r^{*}$ подмножеств $L^{(j)}, 2 \leqslant j \leqslant r+r^{*}$, где

$$
L^{(j)}= \begin{cases}\bigcup_{i=1}^{m_{j}} \overline{X_{i}}, & j=2, \\ \bigcup_{i=1}^{m_{j}} \overline{X_{l+i}}, & l=\sum_{p=2}^{j-1} m_{p}, \quad 3 \leqslant j \leqslant r .\end{cases}
$$

Если $r^{*}>0$, то при каждом $j, r<j \leqslant r+r^{*}$, положим

$$
L^{(j)}=\overline{X_{l+j-r}}, \quad l=\sum_{p=2}^{r} m_{p} .
$$

Указанное разбиение $n$-ода $X$ однозначно определяет связку поворотов $\tau \in P^{0}(X)$ такую, что $T(\tau)=M$. Теорема 6 доказана.

СЛЕДСТВИЕ 7. Каковы бы ни были натуральное число $n \geqslant 3$ и допустимое множество $M$, справедливо следующее неравенство:

$$
\operatorname{card} M \leqslant\left[\frac{-1+\sqrt{9+8 n}}{2}\right],
$$

әде [·] - челая часть числа.

Отметим, что оценка мощности допустимого множества $M$, указанная в следствии 7 , не улучшаема.

Перейдем к рассмотрению простейших отображений дендрита $X \in D$ с произвольным конечньм множеством точек ветвления.

Лемма 5. Пусть $f \in P^{0}(X)$, где $X \in D-$ произвольный дендрит с непустым конечным мнохеством точек ветвления $R(X)$. Тогда $R(X)$ состоит из периодических траекторий отображсения $f$.

ДокАЗАтЕльство. Утверждение леммы 5 справедливо, если сard $R(X)=1$ (следствие 3). Пусть card $R(X) \geqslant 2$. Покажем, что $R(X) \backslash \operatorname{Per}(f)=\varnothing$. Предположим противное. Так как $R(X) \cap \operatorname{Per}(f) \neq \varnothing$ (лемма 2) и $R(X)$ конечно, при сделанном предположении найдутся точки $z^{0} \in R(X) \cap \operatorname{Per}(f)$ периода $m \geqslant 1$ и $z_{1}^{0} \in R(X) \backslash \operatorname{Per}(f)$ такие, что $f^{m}\left(z_{1}^{0}\right)=z^{0}$. Так как $\operatorname{ord} z_{1}^{0} \geqslant 3$, в $X$ существуют две различные невырожденные дуги $\gamma\left(x, z_{1}^{0}\right)$ и $\gamma\left(y, z_{1}^{0}\right)$, имеющие друг с другом и дугой $\gamma\left(z_{1}^{0}, z^{0}\right)$ единственную общую концевую точку $z_{1}^{0}$. Поэтому $\gamma\left(x, z_{1}^{0}\right) \cup \gamma\left(y, z_{1}^{0}\right)$ есть дуга $\gamma(x, y)$. В силу леммы 1 $f^{m}\left(\gamma\left(z^{0}, z_{1}^{0}\right)\right)=\left\{z^{0}\right\}, f^{m}(\gamma(x, y))=\gamma\left(f^{m}(x), f^{m}(y)\right)$, причем $z^{0} \in \gamma\left(f^{m}(x), f^{m}(y)\right)$. 
Отсюда следует, что полньй прообраз $f^{-m}\left(\gamma\left(f^{m}(x), f^{m}(y)\right)\right)$ дуги $\gamma\left(f^{m}(x), f^{m}(y)\right)$ содержит дендрит $\gamma\left(z^{0}, z_{1}^{0}\right) \cup \gamma(x, y)$, гомеоморфньй триоду. Последнее противоречит тому, что $f \in P^{0}(X)$. Лемма 5 доказана.

Пусть множество $T(f \mid R(X))$ периодов периодических точек сужения $f$ на $R(X)$ состоит из натуральных чисел $m_{1}^{0}, \ldots, m_{k}^{0}, k \geqslant 1$, причем $m_{1}^{0}<\cdots<m_{k}^{0}$ при $k>1$. Обозначим через $z^{0}\left(m_{j}^{0}\right)$ точку множества $R(X)$ с периодом $m_{j}, 1 \leqslant j \leqslant k$.

СлЕДСТВИЕ 8. Пусть $f \in P^{0}(X)$, әде $X \in D-$ произвольный дендрит с непустылм конечным множеством точек ветвления $R(X)$. Тогда $m_{1}^{0}<3$.

ДокАЗАТЕЛЬСТво. Предположим, что $m_{1}^{0} \geqslant 3$. В силу леммы 1 и следствия 1

$$
Y=\bigcup_{i=0}^{m_{1}^{0}-2} \gamma\left(z^{i}\left(m_{1}^{0}\right), z^{i+1}\left(m_{1}^{0}\right)\right)
$$

есть инвариантный дендрит с непустьм конечным множеством точек ветвления (здесь $\left.z^{i}\left(m_{1}^{0}\right)=f^{i}\left(z^{0}\left(m_{1}^{0}\right)\right), 0 \leqslant i \leqslant m_{1}^{0}-1\right)$. Тогда непустое множество $R(Y)$ содержит не более чем $m_{1}^{0}-1$ точку. Отсюда, используя лемму 5 , получаем, что в $R(Y) \subseteq R(X)$ существует периодическая точка периода $\leqslant m_{1}^{0}-1$. Последнее невозможно. Следствие 8 доказано.

С каждой точкой $z^{0}\left(m_{j}^{0}\right) \in R(X)$ свяжем континуум $X^{(n)}\left(z^{0}\left(m_{j}^{0}\right)\right) \subset X$, имеющий единственную точку ветвления $z^{0}\left(m_{j}^{0}\right)$ и гомеоморфньй $n$-оду при $n=$ ord $z^{0}\left(m_{j}^{0}\right)$, $1 \leqslant j \leqslant k$. При этом компонента $X_{i}\left(z^{0}\left(m_{j}^{0}\right)\right), 1 \leqslant i \leqslant \operatorname{ord} z^{0}\left(m_{j}^{0}\right)$, множества $X \backslash\left\{z^{0}\left(m_{j}^{0}\right)\right\}$ является также и компонентой множества $X^{(n)}\left(z^{0}\left(m_{j}^{0}\right)\right) \backslash\left\{z^{0}\left(m_{j}^{0}\right)\right\}$, если $R(X) \cap$ $X_{i}\left(z^{0}\left(m_{j}^{0}\right)\right)=\varnothing$. В противном случае компонентой множества $X^{(n)}\left(z^{0}\left(m_{j}^{0}\right)\right) \backslash\left\{z^{0}\left(m_{j}^{0}\right)\right\}$ служит дуга $X_{i}^{(n)}\left(z^{0}\left(m_{j}^{0}\right)\right)$ из $X_{i}\left(z^{0}\left(m_{j}^{0}\right)\right)$ такая, что $\operatorname{card}\left(R(X) \cap \overline{X_{i}^{(n)}\left(z^{0}\left(m_{j}^{0}\right)\right)}\right)=2$, а конщевыми точками $X_{i}^{(n)}\left(z^{0}\left(m_{j}^{0}\right)\right)$ являются точки множества $R(X)$, причем $z^{0}\left(m_{j}^{0}\right)-$ одна из этих точек. В силу леммы 1 , следствий 4 и 5

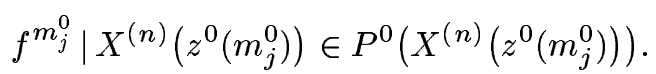

Следовательно, корректно определено допустимое множество $M\left(z^{0}\left(m_{j}^{0}\right)\right)$, связанное с континуумом $X^{(n)}\left(z^{0}\left(m_{j}^{0}\right)\right)$, где $n=n\left(z^{0}\left(m_{j}^{0}\right)\right)=\operatorname{ord} z^{0}\left(m_{j}^{0}\right), 1 \leqslant j \leqslant k$.

ТеОрема 7. Пусть $f \in P^{0}(X)$, где $X \in D-$ произвольный дендрит с непустым конечным множеством $R(X)$. Тогда

1) при $k>1$ для любого $j, 1 \leqslant j \leqslant k-1$, найдется допустимое множество $M\left(z^{0}\left(m_{j}^{0}\right)\right)$, связанное с некоторым континуумом $X^{(n)}\left(z^{0}\left(m_{j}^{0}\right)\right)$, такое, что $m_{j+1}^{0} \in m_{j}^{0} \cdot M\left(z^{0}\left(m_{j}^{0}\right)\right)$;

2) какова бы ни была компонента $X_{i}\left(z^{0}\left(m_{j}^{0}\right)\right)$ множества $X \backslash\left\{z^{0}\left(m_{j}^{0}\right)\right\}$, не содержащая точек ветвления $X$, для произвольной точки $z \in \operatorname{Per}(f) \cap$ $X_{i}\left(z^{0}\left(m_{j}^{0}\right)\right)$ периода $m=m(z)$, где $1 \leqslant j \leqslant k, 1 \leqslant i \leqslant$ ord $z^{0}\left(m_{j}^{0}\right)$, сушествует допустимое множество $M\left(z^{0}\left(m_{j}^{0}\right)\right)$, связанное с некоторым континуумом $X^{(n)}\left(z^{0}\left(m_{j}^{0}\right)\right)$, такое, что $m \in m_{j}^{0} \cdot M\left(z^{0}\left(m_{j}^{0}\right)\right)$. 
ДокАЗАТЕЛЬСТво. Пусть $X^{(n)}\left(z^{0}\left(m_{j}^{0}\right)\right), 1 \leqslant j \leqslant k,-$ произвольньй определенный вьше континуум в $X$. При $m_{j}^{0}=m_{1}^{0}=1 f \mid X^{(n)}\left(z^{0}(1)\right) \in P^{0}\left(X^{(n)}\left(z^{0}(1)\right)\right)$. Тогда множество $T\left(f \mid X^{(n)}\left(z^{0}(1)\right)\right)$ допустимо, и для каждой периодической точки из $X^{(n)}\left(z^{0}(1)\right)$ справедливо утверждение теоремы 7. Рассмотрим случай $m_{j}^{0} \geqslant 2$. Возьмем произвольную точку $z \in \operatorname{Per}(f) \cap X^{(n)}\left(z^{0}\left(m_{j}^{0}\right)\right)$ такую, что $z \notin \gamma\left(z^{p}\left(m_{j}^{0}\right), z^{q}\left(m_{j}^{0}\right)\right)$ при любых $p \neq q, 0 \leqslant p, q \leqslant m_{j}^{0}-1$. Тогда в силу лемм $1,3,5$ и следствия 5 каждая точка $z^{s}=f^{s}(z), 1 \leqslant s \leqslant m_{j}^{0}-1$, обладает следуюшими свойствами:

1) $z^{s} \notin X^{(n)}\left(z^{0}\left(m_{j}^{0}\right)\right)$, но $z^{s} \in X^{(n(s))}\left(z^{s}\left(m_{j}^{0}\right)\right)$, где $n(s)=\operatorname{ord} z^{s}\left(m_{j}^{0}\right)$;

2) $\operatorname{card} I\left(\operatorname{Orb}\left(z^{s}, f^{m_{j}^{0}}\right)\right)=\operatorname{card} I\left(\operatorname{Orb}\left(z, f^{m_{j}^{0}}\right)\right)$.

Свойство 1) влечет за собой выполнение неравенства $m(z) \geqslant m_{j}^{0}$. Так как

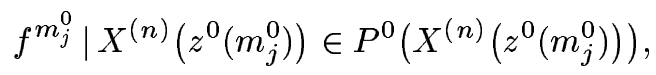

найдется допустимое множество $M\left(z^{0}\left(m_{j}^{0}\right)\right)$ такое, что

$$
\operatorname{card} I\left(\operatorname{Orb}\left(z, f^{m_{j}^{0}}\right)\right) \in M\left(z^{0}\left(m_{j}^{0}\right)\right) .
$$

Используя свойство 2), получаем отсюда, что $m(z) \in m_{j}^{0} \cdot M\left(z^{0}\left(m_{j}^{0}\right)\right)$.

Утверждение 2) теоремы 7 справедливо в силу сказанного вьше. Пусть $k>1,1 \leqslant j \leqslant$ $k-1$. Возьмем произвольную точку $z^{0}\left(m_{j+1}^{0}\right) \in R(X)$. Быть может, найдется континуум $X^{(n)}\left(z^{0}\left(m_{j}^{0}\right)\right)$, содержащий $z^{0}\left(m_{j+1}^{0}\right)$. Так как $m_{j+1}^{0}>m_{j}^{0}$, используя лемму 4 , получаем, что $z^{0}\left(m_{j+1}^{0}\right) \notin \gamma\left(z^{p}\left(m_{j}^{0}\right), z^{q}\left(m_{j}^{0}\right)\right)$ при любых $p \neq q, 0 \leqslant p, q \leqslant m_{j}^{0}-1$. Следовательно, $m_{j+1}^{0} \in m_{j}^{0} \cdot M\left(z^{0}\left(m_{j}^{0}\right)\right)$. Предположим, что не существует континуума $X^{(n)}\left(z^{0}\left(m_{j}^{0}\right)\right)$, содержащего $z^{0}\left(m_{j+1}^{0}\right)$. Тогда каждая дуга $\gamma\left(z^{0}\left(m_{j}^{0}\right), z^{0}\left(m_{j+1}^{0}\right)\right)$ содержит более двух точек из $R(X)$. Выберем дугу $\gamma\left(z^{0}\left(m_{j}^{0}\right), z^{0}\left(m_{j+1}^{0}\right)\right)$ так, чтобы она не содержала точек $\operatorname{Orb}\left(z^{0}\left(m_{j}^{0}\right), f\right)$, отличных от $z^{0}\left(m_{j}^{0}\right)$. Определим инвариантньй относительно $f^{m_{j}^{0}}$ континуум

$$
X^{*}=\bigcup_{s=0}^{s^{*}-1} f^{s m_{j}^{0}}\left(\gamma\left(z^{0}\left(m_{j}^{0}\right), z^{0}\left(m_{j+1}^{0}\right)\right)\right),
$$

здесь $s^{*} m_{j}^{0}$ - наименьшее общее кратное $m_{j}^{0}$ и $m_{j+1}^{0}$. Укажем, что $X^{*}$ либо не содержит точек ветвления, либо имеет единственную точку ветвления $z^{0}\left(m_{j}^{0}\right)$. Кроме того, $f^{m_{j}^{0}} \mid X^{*} \in P^{0}\left(X^{*}\right)$. В силу выбора дуги $\gamma\left(z^{0}\left(m_{j}^{0}\right), z^{0}\left(m_{j+1}^{0}\right)\right)$, леммы 4 и неравенства $m_{j+1}^{0}>m_{j}^{0}$ для произвольной точки $z \in \operatorname{Per}(f) \cap \gamma\left(z^{0}\left(m_{j}^{0}\right), z^{0}\left(m_{j+1}^{0}\right)\right), z \neq z^{0}\left(m_{j}^{0}\right)$, справедливо

$$
I\left(\operatorname{Orb}\left(z, f^{m_{j}^{0}}\right)\right)=I\left(\operatorname{Orb}\left(z^{0}\left(m_{j+1}^{0}\right), f^{m_{j}^{0}}\right)\right), \quad \operatorname{card} I\left(\operatorname{Orb}\left(z, f^{m_{j}^{0}}\right)\right)>1,
$$

где под $I(\cdot)$ понимается индексное множество периодической орбиты относительно $X^{*}$. Пусть точка $z \in R(X) \cap \gamma\left(z^{0}\left(m_{j}^{0}\right), z^{0}\left(m_{j+1}^{0}\right)\right)$ такова, что дуга $\gamma\left(z^{0}\left(m_{j}^{0}\right), z\right)$ не содержит точек ветвления $X$, отличных от $z^{0}\left(m_{j}^{0}\right)$ и $z$. Тогда $\gamma\left(z^{0}\left(m_{j}^{0}\right), z\right) \subset X^{(n)}\left(z^{0}\left(m_{j}^{0}\right)\right)$ и $z \notin \gamma\left(z^{p}\left(m_{j}^{0}\right), z^{q}\left(m_{j}^{0}\right)\right)$ при любых $p \neq q, 0 \leqslant p, q \leqslant m_{j}^{0}-1$. Следовательно, $m(z)=m_{j+1}^{0} \in m_{j}^{0} \cdot M\left(z^{0}\left(m_{j}^{0}\right)\right)$. Теорема 7 доказана.

Теоремы 6,7 , лемма 4 и следствие 8 содержат полную информацию об арифметических соотношениях между периодами периодических точек простейших отображений дендритов из класса $D$ с непустьм конечным множеством точек ветвления. 
СлЕДСТВИЕ 9. Пусть $f \in P^{0}(X)$, где $X \in D-$ произвольный дендрит с непустым конечным мнохеством точек ветвления. Тогда $T(f)$ - ограниченное мнозествво.

Таким образом, если $f \in P^{0}(X)$, а $T(f)$ неограничено, то множество $R(X)$ счетно. Теорема 3 доказана.

4. Доказательство теорем 4 и 5. Начнем с доказательства теоремы 4. Обратим внимание на то, что если множество $R(X)$ счетно, то наряду с периодическими точками отображения $f \in P^{0}(X), R(X)$ может содержать и непериодические точки $f$.

Лемма 6. Пусть $f \in P^{0}(X)$, a $z \in R(X) \backslash \operatorname{Per}(f)$. Тогда не более двух компонент множества $X \backslash\{z\}$ содержат периодические точки $f$.

Действительно, предположив противное, немедленно получаем, что $z \in \operatorname{Per}(f)$.

Обозначим через $\mathrm{Pois}(f)$ множество устойчивых по Пуассону точек отображения $f \in P^{0}(X)$.

Лемма 7. Пусть $f \in P^{0}(X)$, а $x^{0}-$ предельная точка множества $\operatorname{Per}(f)$. Тогда $x^{0} \in \operatorname{Pois}(f)$.

ДокАЗАТЕЛЬСтво. 1) Если существует последовательность $\left\{x_{j}\right\}_{j \geqslant 1} \subset \operatorname{Per}(f)$, сходящаяся к $x^{0}$, и такая, что множество $\left\{m\left(x_{j}\right)\right\}_{j \geqslant 1}$ ограничено, где $m\left(x_{j}\right)$ - (наименьший) период точки $x_{j}$, то в силу непрерьвности $f x^{0} \in \operatorname{Per}(f)$.

2) Будем предполагать, что какова бы ни была последовательность $\left\{x_{j}\right\}_{j \geqslant 1} \subset \operatorname{Per}(f)$, состоящая из попарно различньх точек и сходящаяся к $x^{0}$, множество $\left\{m\left(x_{j}\right)\right\}_{j} \geqslant 1$ неограничено, а $x^{0} \notin \operatorname{Per}(f)$. В силу теоремы 3 множество $R(X)$ счетно. Предположим, что $x^{0} \notin R(X)$. Зафиксируем произвольное $z^{0} \in R(X) \cap \operatorname{Per}(f)$. Тогда найдется компонента $X_{i}\left(z^{0}\right)$ множества $X \backslash\left\{z^{0}\right\}, 1 \leqslant i \leqslant$ ord $z^{0}$, содержашая $x^{0}$. Так как $R(X)-$ замкнутое множество в метрическом пространстве $X$, при сделанном предположении существует окрестность $U\left(x^{0}\right)$ точки $x^{0}$ такая, что саrd $\left(\partial U\left(x^{0}\right)\right) \leqslant 2$ и $U\left(x^{0}\right)$ - невырожденная дуга. Имеем $\left\{x_{j}\right\}_{j \geqslant j_{0}} \subset U\left(x^{0}\right)$ при некотором $j_{0} \geqslant 1$. Возьмем произвольные $j_{1}, j_{2}, j_{2}>j_{1} \geqslant j_{0}$. Предположим для определенности, что $x_{j_{2}} \in \gamma\left(x_{j_{1}}, x^{0}\right)$. Так как в $U\left(x^{0}\right)$ нет точек ветвления $X$, то $\gamma\left(z^{0}, x_{j_{1}}\right) \cup \gamma\left(x_{j_{1}}, x^{0}\right)$ есть дуга $\gamma\left(z^{0}, x^{0}\right)$, содержащая $x_{j_{2}}$. Пусть $m$ - наименьшее общее кратное чисел $m\left(z^{0}\right), m\left(x_{j_{1}}\right)$ и $m\left(x_{j_{2}}\right)$. Тогда ввиду отсутствия точек ветвления в $U\left(x^{0}\right)$ для некоторой дуги $\gamma\left(z^{0}, z\right) \subset \overline{X_{i}\left(z^{0}\right)}$ справедливо $x_{j_{1}}, x_{j_{2}}, x^{0}, f^{m}\left(x^{0}\right) \in \gamma\left(z^{0}, z\right)$, здесь

$$
z= \begin{cases}x^{0}, & \text { если } f^{m}\left(x^{0}\right) \in \gamma\left(x_{j_{2}}, x^{0}\right), \\ f^{m}\left(x^{0}\right), & \text { если } x^{0} \in \gamma\left(x_{j_{2}}, f^{m}\left(x^{0}\right)\right) .\end{cases}
$$

Отсюда получаем, что $\gamma\left(z^{0}, z\right)$ содержит не менее двух точек $\operatorname{Orb}\left(x_{j}, f^{m}\right)$ произвольной точки $x_{j} \in \gamma\left(x_{j_{2}}, x^{0}\right)$. Это противоречит следствию 6 , следовательно, $x^{0} \in R(X)$.

3) Зафиксируем произвольное $\varepsilon>0$. Пусть $\left\{y_{j}\right\}_{j=1}^{p_{0}} \subset \operatorname{Per}(f)$, а $O(Y)=$ $\left\{\operatorname{Orb}\left(y_{j}, f\right)\right\}_{j=1}^{p_{0}}-$ конечная $(\varepsilon / 3)$-сеть компакта $\overline{\operatorname{Per}(f)}$, содержащая не менее двух различных точек. Определим инвариантньй континуум

$$
Y=\bigcup_{y^{\prime}, y^{\prime \prime} \in O(Y)} \gamma\left(y^{\prime}, y^{\prime \prime}\right) .
$$

Тогда множество точек ветвления $Y$ не более чем конечно, и в силу части 2) доказательства $Y \neq X$. Из свойства 3 , леммы 6 и следствия 9 вытекает, что существует компонента 
$U$ открытого множества $X \backslash Y$ и натуральное число $j^{0} \geqslant 1$ такие, что $\left\{x_{j}\right\}_{j \geqslant j} \subset U$, $x^{0} \in U, u=\partial U \in \operatorname{Per}(f) \cap Y$. Последнее вместе с леммой 1 и следствием 4 влечет существование натурального $m=m(\varepsilon)$ такого, что $m$ кратно (наименьшему) периоду точки $u$, и справедливо включение

$$
f^{m}(U) \subset \bar{U} \text {. }
$$

4) Покажем, что для любых точек $u^{\prime}, u^{\prime \prime} \in U \cap \overline{\operatorname{Per}(f)}$ выполнено неравенство

$$
\rho\left(u^{\prime}, u^{\prime \prime}\right)<\varepsilon .
$$

Действительно, пусть точки $y^{\prime}, y^{\prime \prime} \in O(Y)$ таковы, что

$$
\operatorname{diam} \gamma\left(u^{\prime}, y^{\prime}\right) \leqslant \frac{\varepsilon}{3}, \quad \operatorname{diam} \gamma\left(u^{\prime \prime}, y^{\prime \prime}\right) \leqslant \frac{\varepsilon}{3},
$$

где $\operatorname{diam}(\cdot)$ - диаметр дуги. В частности, $\rho\left(u, u^{\prime}\right) \leqslant \varepsilon / 3, \rho\left(u, u^{\prime \prime}\right) \leqslant \varepsilon / 3$, так как $u \in \gamma\left(u^{\prime}, y^{\prime}\right) \cap \gamma\left(u^{\prime \prime}, y^{\prime \prime}\right)$. Поэтому $\rho\left(u^{\prime}, u^{\prime \prime}\right) \leqslant 2 \varepsilon / 3<\varepsilon$. Тогда, используя (3) и (4), получаем, что для любого $\varepsilon>0$ существует $m=m(\varepsilon)$ такое, что $\rho\left(x^{0}, f^{m}\left(x^{0}\right)\right)<\varepsilon$, т.е. $x^{0} \in \operatorname{Pois}(f)$. Лемма 7 доказана.

СледСТвИЕ 10. Пусть $f \in P^{0}(X)$, a $x^{0}-$ предельная точка множества $\operatorname{Per}(f)$. Тогда $x^{0} \in \operatorname{Per}(f)$.

ДокАЗАТЕльство. Предположим, что $x^{0} \notin \operatorname{Per}(f)$. Тогда для любой последовательности $\left\{x_{j}\right\}_{j \geqslant 1} \subset \operatorname{Per}(f)$, состоящей из попарно различных точек и сходящейся к $x^{0}$, справедливо, что $\left\{m\left(x_{j}\right)\right\}_{j \geqslant 1}$ - неограниченное множество. В силу леммы $7 x^{0} \in R(X) \cap$ Pois $(f)$. Отсюда, используя инвариантность $R(X)$, получаем, что $\omega\left(x^{0}, f\right) \subset R(X)$, $\omega\left(x^{0}, f\right)$ замкнуто и плотно в себе. Следовательно, любая точка из $\omega\left(x^{0}, f\right)$ есть точка конденсации $[19$, с. 260, с. 425$]$. Последнее невозможно, так как счетное множество $R(X)$ не содержит точек конденсации. Следствие 10 доказано.

Таким образом, утверждение 1) теоремы 4 справедливо. Перейдем к доказательству утверждений 2) и 3). Будем предполагать, что $\operatorname{Per}(f) \neq X($ если $\operatorname{Per}(f)=X$, то утверждения 2) и 3 ) справедливы). Обозначим через $V$ произвольную компоненту открытого множества $X \backslash \operatorname{Per}(f)$.

Лемма 8. Если $f \in P^{0}(X)$, то существует дуга $\gamma(x, y) \subset \bar{V}$ такая, что $x \in \operatorname{Per}(f)$ u $f^{m}(\gamma(x, y)) \subseteq \gamma(x, y)$ при некотором натуральном $m$, кратном периоду точки $x$.

ДокАЗАТЕЛЬСтво. Заметим, что в силу леммы $6 \operatorname{card}(\operatorname{Per}(f) \cap \bar{V})=1$ или 2. Если $\operatorname{card}(\operatorname{Per}(f) \cap \bar{V})=2$, то утверждение леммы 8 следует из леммы 1 .

Пусть теперь $x$ - единственная точка множества $\operatorname{Per}(f) \cap \bar{V}$. В силу леммы 1 , следствий 4 и 5 существует натуральное число $m \geqslant 1$, кратное периоду точки $x$ и такое, что $f^{m}(\bar{V}) \subseteq \bar{V}$. Предположим, что $\bar{V}$ не содержит дуг, инвариантных относительно отображения $f^{m}$. Возьмем произвольную концевую точку $y \neq x$ множества $\bar{V}$. При сделанном предположении $f^{m}(y) \notin \gamma(x, y)$ и существуют точки $y_{1} \in R(X) \cap \gamma(x, y) \cap \gamma\left(x, f^{m}(y)\right)$ и $y_{2} \in \gamma\left(x, y_{1}\right)$ такие, что $f^{m}\left(y_{2}\right)=y_{1}$. Тогда при любом $j \geqslant 1 \gamma\left(x, y_{1}\right) \subseteq f^{m j}\left(\gamma\left(x, y_{1}\right)\right)$,

$$
\rho\left(x, \omega\left(y_{1}, f^{m}\right)\right) \geqslant \frac{d}{2}, \quad \text { где } d=\operatorname{diam} \gamma\left(x, y_{1}\right) \text {. }
$$


$\mathrm{C}$ другой стороны, так как $y_{1} \in R(X)$, множество $\omega\left(y_{1}, f^{m}\right)$ не более чем счетно и $\omega\left(y_{1}, f^{m}\right) \cap \operatorname{Per}(f) \neq \varnothing$. В силу включения $\omega\left(y_{1}, f^{m}\right) \subset \bar{V}$ справедливо равенство $\omega\left(y_{1}, f^{m}\right) \cap \operatorname{Per}(f)=\{x\}$. Последнее противоречит неравенству (5). Лемма 8 доказана.

Справедливость утверждений 2) и 3) теоремы 4 вытекает из леммы 8 и локальной связности $X$.

Утверждение 4) теоремы 4 является следствием полученных результатов. Действительно, положим для любых $x, y \in \operatorname{Per}(f)$

$$
\rho_{m i}(x, y)=\max _{0 \leqslant j \leqslant i} \rho\left(f^{m j}(x), f^{m j}(y)\right) .
$$

В силу леммы 7 и следствия 6 справедлива

Лемма 9. Пусть $f \in P^{0}(X)$, a $\varepsilon$ - произвольное полохительное число. Тогда

1) существует конечная $\varepsilon$-сеть $\left\{\operatorname{Orb}\left(y_{s}, f\right)\right\}_{s=1}^{p} \subset \operatorname{Per}(f)$ компакта $\operatorname{Per}(f)$ в

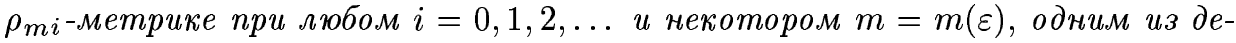
лителей которого служит наименьшее общее кратное периодов $m\left(y_{1}\right), \ldots$, $m\left(y_{p}\right)$ точек $y_{1}, \ldots, y_{p}$ соответственно;

2) каково би ни было $\varepsilon_{1}, 0<\varepsilon_{1}<\varepsilon$, существует конечная $\varepsilon_{1}$-сеть $\left\{\operatorname{Orb}\left(y_{s}, f\right)\right\}_{s=1}^{p_{1}}$ $\subset \operatorname{Per}(f)\left(p_{1} \geqslant p\right)$ компакта $\operatorname{Per}(f)$ в $\rho_{m i}$-метрике при любом $i=0,1,2, \ldots$ $u m\left(\varepsilon_{1}\right)=m(\varepsilon)=m$.

Обозначим через $r_{m i}(\varepsilon)$ минимальное число элементов в конечной $\varepsilon$-сети компакта $\operatorname{Per}(f)$, снабженного $\rho_{m i}$-метрикой. Тогда в силу леммы 9 , работы [20] и утверждений 1), 3) теоремы 4 справедливы равенства

$$
m h(f)=h\left(f^{m}\right)=h\left(f^{m} \mid \Omega\left(f^{m}\right)\right)=h\left(f^{m} \mid \operatorname{Per}(f)\right)=\lim _{\varepsilon \rightarrow 0} \varlimsup_{i \rightarrow+\infty} \frac{1}{m i} \ln r_{m i}(\varepsilon)=0 .
$$

Теорема 4 доказана полностью.

Приведем утверждение, играющее важную роль в понимании структуры простейших отображений.

СлЕдСТВИЕ 11. Если $X$ - это $n$-од $(n \geqslant 3)$, то для любого отображения $f \in P^{0}(X)$ существует связка поворотов $\tau \in P^{0}(X)$ такая, что сужение $f \mid \Omega(f)$ топологически сопряжено сужению $\tau$ на некоторое замкнутое инвариантное относительно $\tau$ подмножество $X$.

Пусть $m$ - произвольное натуральное число, $p$ - произвольное простое число, а $X$ дендрит из примера 2. Сохраним обозначения этого примера и укажем отображения $f_{1}, f_{2} \in P^{0}(X)$ такие, что $T\left(f_{1}\right)=\{1, m j\}_{j \geqslant 1}, T\left(f_{2}\right)=\left\{p^{j}\right\}_{j \geqslant 0}$.

ДОКАЗАТЕЛЬСТВО ТЕОРЕМЫ 5 . Положим в случае 1 )

$$
n_{j}= \begin{cases}3, & \text { если } j=0, m=1 \text { или } 2, \\ m, & \text { если } j=0, m \geqslant 3, \\ 2+j, & \text { если } j \geqslant 1, m=1, \\ m(j+1), & \text { если } j \geqslant 1, m \geqslant 2,\end{cases}
$$

в случае 2)

$$
n_{j}= \begin{cases}3, & \text { если } j=0, p=2, \\ p, & \text { если } j=0, p>2, \\ p^{j+1}, & \text { если } j \geqslant 1, p \geqslant 2 .\end{cases}
$$


Определим отображение $f_{i} \in P^{0}(X)(i=1,2)$, полагая $f_{i}\left|X^{(0)}=\mathrm{id}\right| X^{(0)}$; при $j=0, m=1$ или $2, p=2 f_{i} \mid X^{\left(n_{0}\right)}=\tau$, где $\tau: X^{\left(n_{0}\right)} \rightarrow X^{\left(n_{0}\right)}-$ связка поворотов такая, что $T(\tau)=\{1,2\}$; при $j=0, m, p \geqslant 3 f_{i} \mid X^{\left(n_{0}\right)}$ - поворот $X^{\left(n_{0}\right)}$ на угол $2 \pi / n_{0}$; при любых $j, m \geqslant 1, p \geqslant 2 f_{i} \mid X^{\left(n_{j}\right)}$ - поворот $X^{\left(n_{j}\right)}$ в плоскости $x_{3}=1 / j$ на угол $2 \pi / n_{j}$. Тогда $T\left(f_{1}\right)=\{1, m j\}_{j \geqslant 1}, T\left(f_{2}\right)=\left\{p^{j}\right\}_{j} \geqslant 0$. В силу утверждения 4) теоремы $4 h\left(f_{1}\right)=h\left(f_{2}\right)=0$. Теорема 5 доказана.

\section{СПИСОК ЦИТИРОВАННОЙ ЛИТЕРАТУРЫ}

[1] Шарковский А. Н. Сосуществование циклов непрерьвного отображения прямой в себя // Укр. матем. ж. 1964. Т. 16. №1. С. 61-71.

[2] Misiurewicz M. Horseshoes for mappings of the interval // Bull. Acad. Polon. Sci. Ser. Sci. Math. 1979. V. 27. №2. P. 167-169.

[3] Блох А. М. О "спектральном разложении" для кусочно-монотонных отображений отрезка // УМН. 1982. Т. 37. № 3. С. 175-176.

[4] Ефремова Л. С. Периодические орбиты и степень непрерывного отображения окружности // Дифференциальные и интегральные уравнения. Вып. 2. Горький: ГГУ, 1978. С. 109-115.

[5] Ефремова Л. С., Рахманкулов Р. Г. Теоремы сосуществования периодических орбит эндоморфизмов окружности // Дифференциальные и интегральные уравнения. Вып. 4. Горький: ГГУ, 1980. С. 116-118.

[6] Ефремова Л.С. Периодические точки непрерьвного отображения окружности // IX Международная конференция по нелинейным колебаниям (Киев, 30.08.81-06.09.81). Т. 2. Киев: ИМ АН УССР, 1984. С. 124-126.

[7] Малкин М.И. Периодические орбиты, энтропия и множества вращения непрерывных отображений окружности // Укр. матем. ж. 1983. Т. 35. №3. С. 327-332.

[8] Block L., Guckenheimer J., Misiurewicz M., Young L.S. Periodic points and topological entropy of one-dimensional maps // Lecture Notes in Math. 1980. V. 819. P. 18-34.

[9] Bernhardt C. Periodic orbits of continuous mappings of the circle without fixed points // Ergodic Theory Dynam. Systems. 1981. V. 1. № 4. P. 413-417.

[10] Alseda L., Llibre J. A note on the set of periods for continuous maps of the circle which have degree one // Proc. Amer. Math. Soc. 1985. V. 93. №1. P. 133-138.

[11] Baldwin St. An extension of Šarkovskii's theorem to the $n$-od // Ergodic Theory Dynam. Systems. 1991. V. 11. № 2. P. 249-271.

[12] Baldwin St. Some limitations toward extending Šarkovskii's theorem to connected linearly ordered spaces // Houston J. Math. 1991. V. 17. №1. P. 39-53.

[13] Alseda L., Llibre J., Misiurewicz M. Periodic orbits of maps of $Y / /$ Trans. Amer. Math. Soc. 1989. V. 313. № 2. P. 475-538.

[14] Llibre J., Misiurewicz M. Horseshoes, entropy and periods for graph maps // Topology. 1993. V. 32. P. 649-664.

[15] Blokh A. M. Periods implying almost all periods, trees with snowflakes, and zero entropy maps // Nonlinearity. 1992. V. 5. P. 1375-1382.

[16] Куратовский К. Топология. Т. 2. М.: Мир, 1969.

[17] Ayres W. L. Some generalization of the Scherrer fixed point theorem // Fund. Math. 1930. V. 16. P. 332-336.

[18] Шарковский А.Н. О притягивающих и притягивающихся множествах // Докл. АН CCCP. 1965. T. 160. № 5. C. 1036-1038.

[19] Куратовский К. Топология. Т. 1. М.: Мир, 1966.

[20] Динабург Е.И. Связь между различными энтропийными характеристиками динамических систем // Изв. АН СССР. Сер. матем. 1971. Т. 35. № 2. С. 324-366.

Нижегородский государственньй университет им. Н. И. Лобачевского

Поступило

E-mail : efr@mm.unn.ac.ru

11.04 .94

Исправленный вариант

27.08 .96 